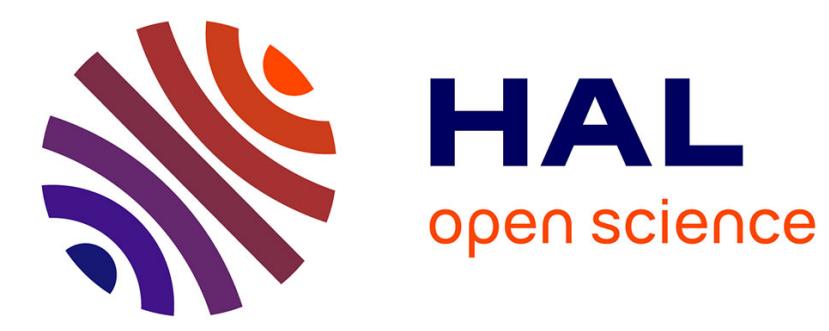

\title{
Link between copula and tomography
}

Doriano-Boris Pougaza, Ali Mohammad-Djafari, Jean-François Bercher

\section{To cite this version:}

Doriano-Boris Pougaza, Ali Mohammad-Djafari, Jean-François Bercher. Link between copula and tomography. Pattern Recognition Letters, 2010, 31 (14), pp.2258-2264. 10.1016/j.patrec.2010.05.001 . hal-00509705

\section{HAL Id: hal-00509705 \\ https://hal.science/hal-00509705}

Submitted on 14 Aug 2010

HAL is a multi-disciplinary open access archive for the deposit and dissemination of scientific research documents, whether they are published or not. The documents may come from teaching and research institutions in France or abroad, or from public or private research centers.
L'archive ouverte pluridisciplinaire HAL, est destinée au dépôt et à la diffusion de documents scientifiques de niveau recherche, publiés ou non, émanant des établissements d'enseignement et de recherche français ou étrangers, des laboratoires publics ou privés. 


\title{
Link between Copula and Tomography
}

\author{
Doriano-Boris Pougaza ${ }^{\mathrm{a}}$, Ali Mohammad-Djafari ${ }^{\mathrm{a}}$, Jean-François Bercher ${ }^{\mathrm{a}, \mathrm{b}}$ \\ ${ }^{a}$ Laboratoire des Signaux et Systèmes \\ UMR 8506 (CNRS-SUPELEC-Univ Paris Sud 11) \\ SUPELEC, Plateau de Moulon \\ 3 rue Joliot Curie, 91192 Gif-sur-Yvette Cedex, France \\ ${ }^{b}$ Laboratoire d'Informatique Gaspard Monge, \\ UMR 8049, CNRS-ESIEE-Université Paris-Est, \\ 5 bd Descartes, 77454 Marne-la-Vallée Cedex 2, France
}

\begin{abstract}
An important problem in statistics is to determine a joint probability distribution from its marginals and an important problem in Computed Tomography $(\mathrm{CT})$ is to reconstruct an image from its projections. In the bivariate case, the marginal probability density functions $f_{1}(x)$ and $f_{2}(y)$ are related to their joint distribution $f(x, y)$ via horizontal and vertical line integrals. Interestingly, this is also the case of a very limited angle $\mathrm{X}$ ray $\mathrm{CT}$ problem where $f(x, y)$ is an image representing the distribution of the material density and $f_{1}(x), f_{2}(y)$ are the horizontal and vertical line integrals. The problem of determining $f(x, y)$ from $f_{1}(x)$ and $f_{2}(y)$ is an ill-posed undetermined inverse problem. In statistics the notion of copula is exactly introduced to characterize all the possible solutions to the problem of reconstructing a bivariate density from its marginals. In this paper, we elaborate on the possible link between Copula and CT and try to see whether we can use the methods used in one domain into the other.
\end{abstract}

Key words: Copula, Tomography, Joint and marginal distributions, Image reconstruction, Additive and Multiplicative Backprojection, Maximum Entropy, Archimedian Copulas.

\section{Introduction}

The word copula originates from the Latin meaning link, chain, union. In statistical literature, according to the seminal result in the copula's the4 ory stated by Abe Sklar [1] in 1959, a copula is a function that connects 5 a multivariate distribution function to its univariate marginal distributions. 6 There is an increasing interest concerning copulas, widely used in Financial 
Mathematics and in modelling of Environmental Data [2, 3]. Recently, in Computational Biology, copulas were used for DNA analysis [4]. Copula appears to be a powerful tool to model the structure of dependence $[5,6]$. Copulas are useful for constructing joint distributions, particularly with nonGaussian random variables [7].

In $2 \mathrm{D}$ case, interpreting the joint probability density function $f(x, y)$ as an image and its marginal probability densities $f_{1}(x)$ and $f_{2}(y)$ as horizontal and vertical line integrals:

$$
f_{1}(x)=\int f(x, y) d y \text { and } f_{2}(y)=\int f(x, y) d x
$$

we see that the problem of determining $f(x, y)$ from $f_{1}(x)$ and $f_{2}(y)$ is an ill-posed (inverse) problem [8-10]. It is a well known fact that while a distribution has a unique set of marginals, the converse is not necessarily true. That is, many distributions may share a common subset of marginals. In general, it is not possible to uniquely reconstruct a distribution from its marginals. This is illustrated in Figure 1: Figure 1 (a) shows the forward problem given by (1), whereas Figure 1 (b) illustrates the inverse problem. As we will see later, all functions in the form of

$$
f(x, y)=f_{1}(x) f_{2}(y) c\left(F_{1}(x), F_{2}(y)\right)
$$

where $F_{1}(x), F_{2}(y)$ are the marginal cumulative distributions functions (cdf's) and $c$ is any copula density function, are solutions of this problem. Interestingly, this is very similar to the probability density function (pdf) reconstruction problem considered in [11], where a special copula was designed. The approach in [11] could certainly be interpreted using the results presented here.

In 1917, Johann Radon introduced the Radon transform (RT) [12, 13] which was later used in CT [14]. Indeed, if we denote by $f(x, y)$, the spatial distribution of the material density in a section of the body, a very simple model that relates a line of the radiography image $p(r, \theta)$ at direction $\theta$ to $f(x, y)$ is given by the Radon transform:

$$
p(r, \theta)=\int_{L_{r, \theta}} f(x, y) d l=\iint_{\mathbb{R}^{2}} f(x, y) \delta(r-x \cos \theta-y \sin \theta) d x d y,
$$

where $L_{r, \theta}=\{(x, y): r=x \cos \theta+y \sin \theta\}$ and $\delta$ is the Dirac's delta function. The experimental setup is presented in Figure 2.

If now we consider only the horizontal $\theta=0$ projection and the vertical $\theta=\pi / 2$ projection, we see easily the connexion between these two problems. 


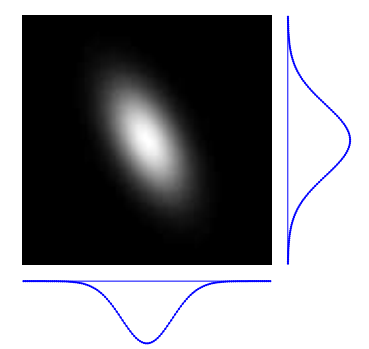

Forward problem: Given $f(x, y)$ compute $f_{1}(x)$ and $f_{2}(y)$

(a)

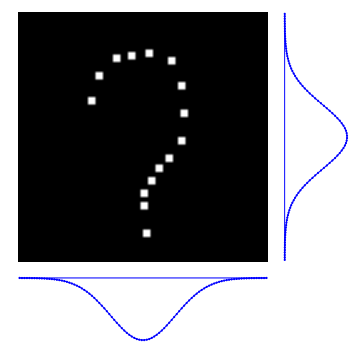

Inverse problem:

determine $f(x, y)$

(b)

Figure 1: Forward and inverse problems

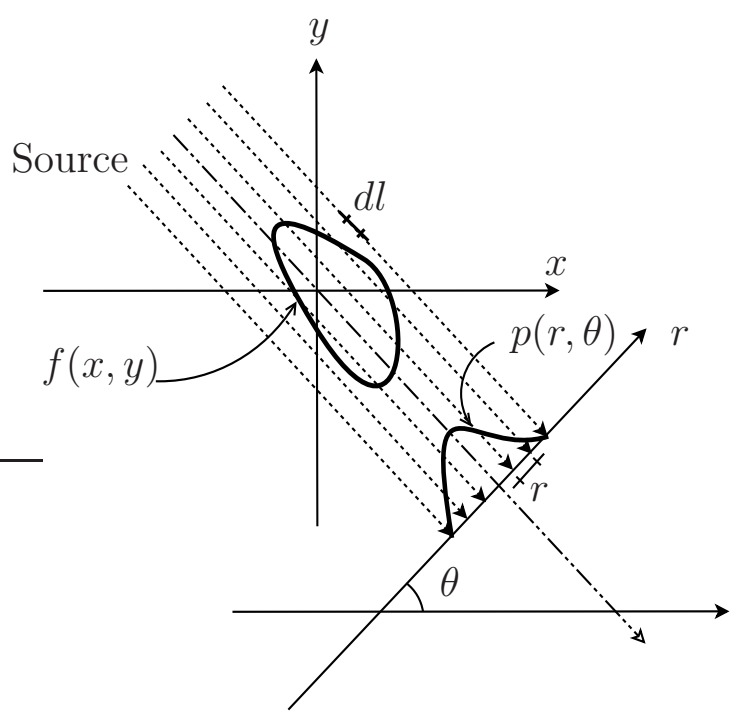

Figure 2: X ray Computed Tomography: 2D parallel geometry.

The main object of this paper is to explore in more details these relations, and exploit the similarity between the two problems as a new approach to image reconstruction in Computed Tomography.

The rest of this paper is organized as follows: In section 2, we present a summary of the necessary definitions and properties of copulas and highlight methods to generate a copula. In section 3 , we present the main tomographic image reconstruction methods based on the Radon inversion formula. In sec- 
tion 4, we will be in the heart of the link and relations between the notions of these two previous sections. Section 5 and 6 are devoted to details concerning our method. Some preliminary results from our Copula-Tomography Matlab package are shown.

\section{Copula}

In this section, we give a few definitions and properties of copulas that we need in the rest of the paper. For more details about this section we refer to [15]. First, we note by $F(x, y)$ a bivariate cumulative distribution function (cdf), by $f(x, y)$ its bivariate probability density function (pdf), by $F_{1}(x)$, $F_{2}(y)$ its marginal cdf's and $f_{1}(x), f_{2}(y)$ their corresponding pdf's with their classical relations :

$$
\begin{aligned}
F(x, y) & =\int_{-\infty}^{x} \int_{-\infty}^{y} f(s, t) \mathrm{d} s \mathrm{~d} t, \quad f(x, y)=\frac{\partial^{2} F(x, y)}{\partial x \partial y}, \\
F_{1}(x) & =\int_{-\infty}^{x} f_{1}(s) \mathrm{d} s=F(x, \infty), \quad F_{2}(y)=\int_{-\infty}^{y} f_{2}(t) \mathrm{d} t=F(\infty, y), \\
f_{1}(x) & =\frac{\mathrm{d} F_{1}(x)}{\mathrm{d} x}=\int f(x, y) \mathrm{d} y, \quad f_{2}(y)=\frac{\mathrm{d} F_{2}(y)}{\mathrm{d} y}=\int f(x, y) \mathrm{d} x .
\end{aligned}
$$

Definition Bivariate Copula: A bivariate copula, or shortly a copula is a function $C$ from $[0,1]^{2}$ to $[0,1]$ with the following properties:

- $\forall u, v \in[0,1], C(u, 0)=0=C(0, v)$,

- $\forall u, v \in[0,1], C(u, 1)=u$ and $C(1, v)=v$ and

- $C\left(u_{2}, v_{2}\right)-C\left(u_{2}, v_{1}\right)-C\left(u_{1}, v_{2}\right)+C\left(u_{1}, v_{1}\right) \geq 0$

for all $u_{1}, u_{2}, v_{1}, v_{2} \in[0,1]$ such that $u_{1} \leq u_{2}, v_{1} \leq v_{2}$.

Theorem 2.1. Sklar's Theorem (for proof, see [16]): Let $F$ be a two-dimensional distribution function with marginal distributions functions $F_{1}$ and $F_{2}$. Then there exists a copula $C$ such that:

$$
F(x, y)=C\left(F_{1}(x), F_{2}(y)\right) .
$$

Conversely, for any univariate distribution functions $F_{1}$ and $F_{2}$ and any copula $C$, the function $F$ is a two-dimensional distribution function with marginals $F_{1}$ and $F_{2}$, given by (4).

Lemma 2.2. If the marginal functions are continuous, then the copula $C$ is unique, and is given by

$$
C(u, v)=F\left(F_{1}^{-1}(u), F_{2}^{-1}(v)\right) .
$$


Definition Copula Density: From (4) and differentiating (5) gives the density of a copula

$$
c(u, v)=\frac{\partial^{2} C}{\partial u \partial v}=\frac{f\left(F_{1}^{-1}(u), F_{2}^{-1}(v)\right)}{f_{1}\left(F_{1}^{-1}(u)\right) f_{2}\left(F_{2}^{-1}(v)\right)},
$$

and thus

$$
f(x, y)=f_{1}(x) f_{2}(y) c\left(F_{1}(x), F_{2}(y)\right) .
$$

An usual simple example is the product or independent copula:

$$
\Pi(u, v)=u v \longrightarrow c(u, v)=1, \quad(u, v) \in[0,1]^{2} .
$$

Property 2.3. Any copula $C(u, v)$, satisfies the inequality

$$
W(u, v) \leq C(u, v) \leq M(u, v),
$$

where the Fréchet-Hoeffding upper bound copula $M(u, v)$ (or comonotonicity copula) is :

$$
M(u, v)=\min (u, v),
$$

and the Fréchet-Hoeffding lower bound $W(u, v)$ (or countermonotonicity copula) is:

$$
W(u, v)=\max \{u+v-1,0\}, \quad(u, v) \in[0,1]^{2} .
$$

Generating Copulas by the Inversion Method: A straightforward method is based directly on Sklar's theorem. Given $F(x, y)$ the joint cdf of two random variables $X, Y$ and $F_{1}(x)$ and $F_{2}(y)$ their marginal cdf's, all assumed to be continuous. The corresponding copula can be constructed by using the unique inverse transformations (Quantile transform) $x=F_{1}^{-1}(u)$, $y=F_{2}^{-1}(v)$, and the equation (5) where $u, v$ are uniform on $[0,1]$.

\subsection{Archimedean Copulas}

The Archimedean copulas (see [15] page 109) form an important class of copulas which generalise the usual copulas.

Theorem 2.4. Let $\varphi$ be a continuous, strictly decreasing function from $[0,1]$ to $[0, \infty]$ such that $\varphi(1)=0$, and let $\varphi^{[-1]}$ be the pseudo-inverse ${ }^{1}$ of $\varphi$. Let $C$ be the function from $[0,1]^{2}$ to $[0,1]$ given by

$$
C(u, v)=\varphi^{[-1]}(\varphi(u)+\varphi(v)) .
$$

Then $C$ is a copula if and only if $\varphi$ is convex.

$$
{ }^{1} \varphi^{[-1]}(t)= \begin{cases}\varphi^{-1}(t), & 0 \leq t \leq \varphi(0) \\ 0, & \varphi(0) \leq t \leq \infty\end{cases}
$$


Archimedean copulas are in the form (12) and the function $\varphi$ is called the generator of the copula. $\varphi$ is a strict generator if $\varphi(0)=\infty$, then $\varphi^{[-1]}=\varphi^{-1}$ and

$$
C(u, v)=\varphi^{-1}(\varphi(u)+\varphi(v)) .
$$

\section{Property 2.5. Any Archimedean copula $C$ satisfies the following algebraic} properties:

- $C(u, v)=C(v, u)$ meaning that $C$ is symmetric;

- $C(C(u, v), w)=C(u, C(v, w))$;

- If $a>0$, then a $\varphi$ is again a generator of $C$.

There are many families of Archimedean copulas constructed from different generators $\varphi_{\alpha}$ with a suitable parameter $\alpha$.

For example $\varphi_{\alpha}(t)=\frac{1}{\alpha}\left(t^{-\alpha}-1\right)$ and $\varphi_{\alpha}(t)=\ln (1-\alpha \ln t)$ yield successively to Clayton copula $C_{\alpha}(u, v)=\left[\max \left(u^{-\alpha}+v^{-\alpha}-1,0\right)\right]^{-1 / \alpha}$ and GumbelHougaard copula $C_{\alpha}(u, v)=u v \exp (-\alpha \ln u \ln v)$.

\section{Tomography}

In $2 \mathrm{D}$, the mathematical problem of tomography is to determine the bivariate function $f(x, y)$ from its line integrals $p(r, \theta)$ (see Eq.(3)). Radon has shown that this problem has a unique solution if we know $p(r, \theta)$ for all $\theta \in[0, \pi]$ and all $r \in \mathbb{R}$, then $f(x, y)$ can be computed by the inverse Radon transform (for details, see [17] ) :

$$
f(x, y)=\left(-\frac{1}{2 \pi^{2}}\right) \int_{0}^{\pi} \int_{-\infty}^{+\infty} \frac{\frac{\partial p(r, \theta)}{\partial r}}{r-x \cos \theta-y \sin \theta} \mathrm{d} r \mathrm{~d} \theta
$$

However, if the number of projections is limited, then the problem is ill-posed and the problem has an infinite number of solutions.

To present briefly the main classical methods in CT, we start by decomposing the inverse RT in the following parts:

$$
\text { Derivative } \mathcal{D}: \quad \bar{p}_{\theta}(r)=\frac{\partial p(r, \theta)}{\partial r},
$$

$$
\text { Hilbert Transform } \mathcal{H}: \quad \widetilde{\bar{p}}\left(r^{\prime}, \theta\right)=\frac{1}{\pi} \text { p.v. } \int_{-\infty}^{+\infty} \frac{\bar{p}(r, \theta)}{r-r^{\prime}} \mathrm{d} r
$$

where p.v. is the Cauchy principal value.

Backprojection $\mathcal{B}: f(x, y)=\frac{1}{2 \pi} \int_{0}^{\pi} \widetilde{\bar{p}}\left(r^{\prime}=x \cos \theta+y \sin \theta, \theta\right) \mathrm{d} \theta$. 
Then defining the one dimensional inverse Fourier transform $\mathcal{F}_{1}^{-1}$ by

$$
\text { Inverse Fourier } \mathcal{F}_{1}^{-1}: P(\Omega, \theta)=\int p(r, \theta) \exp [j \Omega r] \mathrm{d} r \text {. }
$$

Using the properties of the Fourier transform $\mathcal{F}_{1}$ and the derivative $\mathcal{D}$, from (15) we have:

$$
\bar{P}(\Omega, \theta)=\Omega P(\Omega, \theta),
$$

the relation between $\mathcal{H}$ and $\mathcal{F}_{1}$ yields :

$$
\widetilde{\bar{P}}(\Omega, \theta)=\operatorname{sgn}(\Omega) \bar{P}(\Omega, \theta)=\operatorname{sgn}(\Omega) \Omega P(\Omega, \theta)=|\Omega| P(\Omega, \theta) .
$$

Finally the filtered backprojection which is currently the most used reconstruction method is performed by the following formula:

$$
f(x, y)=\mathcal{B} \mathcal{H} \mathcal{D} p(r, \theta)=\mathcal{B} \mathcal{F}_{1}^{-1}|\Omega| \mathcal{F}_{1} p(r, \theta)
$$

that is

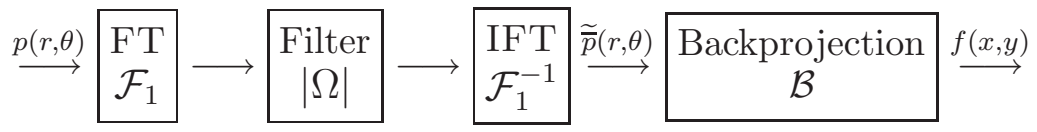

In X-ray $\mathrm{CT}$, if we have a great number of projections uniformly distributed over the angles interval $[0, \pi]$, the filtered backprojection (FBP) or even the simple backprojection (BP) image are good solutions to the inverse CT problem [18]. But, when we are restricted to only two projections, the FBP or BP images are not correct reconstruction [19-21].

\section{Link between Copula and Tomography}

Now, let consider the particular case where we have only two projections $\theta=0$ and $\theta=\pi / 2$. Then

$$
\begin{aligned}
p_{0}(r) & =\iint f(x, y) \delta(r-x) \mathrm{d} x \mathrm{~d} y=\int f(r, y) \mathrm{d} y, \\
p_{\pi / 2}(r) & =\iint f(x, y) \delta(r-y) \mathrm{d} x \mathrm{~d} y=\int f(x, r) \mathrm{d} x
\end{aligned}
$$

and if we let $f_{1}=p_{0}$ and $f_{2}=p_{\pi / 2}$ we can deduce the following new methods, inspired by the reconstruction approaches in CT, for the inverse problem that consists in determining the probability density $f(x, y)$ from its marginals $f_{1}(x)$ and $f_{2}(y)$ : 
Backprojection:

$$
f(x, y)=\frac{1}{2}\left(f_{1}(x)+f_{2}(y)\right) .
$$

Filtered Backprojection:

$$
f(x, y)=\frac{1}{2}\left(\int \frac{\frac{\partial f_{1}}{\partial x}\left(x^{\prime}\right)}{x^{\prime}-x} \mathrm{~d} x^{\prime}+\int \frac{\frac{\partial f_{2}}{\partial y}\left(y^{\prime}\right)}{y^{\prime}-y} \mathrm{~d} y^{\prime}\right)
$$

which can also be implemented in the Fourier domain as it follows

$$
\begin{aligned}
f(x, y) & =\frac{1}{2} \int e^{+j \xi x}|\xi|\left(\int e^{-j \xi x^{\prime}} f_{1}\left(x^{\prime}\right) \mathrm{d} x^{\prime}\right) \mathrm{d} \xi \\
& +\frac{1}{2} \int e^{+j \nu y}|\nu|\left(\int e^{-j \nu y^{\prime}} f_{2}\left(y^{\prime}\right) \mathrm{d} y^{\prime}\right) \mathrm{d} \nu .
\end{aligned}
$$

\section{How to use Copula in Tomography}

The definition and the notion of copula give us the possibility to propose new X ray CT methods. Let first consider the case of two projections. In this case, immediately, we can propose a first use which corresponds to the case of independent copula, as given in (8). We call this method Multiplicative Backprojection (MBP) (see [22])

MBP:

$$
f(x, y)=f_{1}(x) f_{2}(y)
$$

If we compare the equation (19) to (21) instead of the classical BP which is an additive operation or Additive Backprojection, the name MBP comes naturally. In Figure 3 we give comparisons of BP and MBP. As we can see on the image original 1, at least the image obtained by MBP is better than the one obtained by $\mathrm{BP}$ and it satisfies exactly the marginals.

We may still do better if we choose another copula rather than the independent copula, by proposing the following method that we call Copula Backprojection (CopBP).

\section{CopBP:}

$$
f(x, y)=f_{1}(x) f_{2}(y) c\left(F_{1}(x), F_{2}(y)\right)
$$

where $c(u, v)$ is a parametrized copula.

Here the main question is how to choose an appropriate copula for the particular application. This problem can be thought as a way to introduce some prior information, just enough to choose an appropriate family of copula. For example if we know that the joint density has only one mode, and 
can be approximated by a bivariate Gaussian, $\Phi^{-1}$ denoting the inverse of the standard Gaussian cdf, then we can use a Gaussian copula whose expression is given by

$$
C_{\rho}(u, v)=\frac{A}{2 \pi} \int_{-\infty}^{\Phi^{-1}(u)} \int_{-\infty}^{\Phi^{-1}(v)} \exp \left\{\frac{-\left(s^{2}-2 \rho s t+t^{2}\right)}{2\left(1-\rho^{2}\right)}\right\} d s d t
$$

where $A=\left(1-\rho^{2}\right)^{-1 / 2}$ and $\rho=0$ correspond to copulas $\Pi(u, v)$ in Eq. (8) and where $\rho=-1,+1$ give respectively the copulas $W(u, v)$ and $M(u, v)$ in Equations (11) and (10). The corresponding Gaussian copula density is:

$$
c_{\rho}(u, v)=A \exp \left\{\frac{-A^{2}}{2}\left(\left(\rho \Phi^{-1}(u)\right)^{2}-2 \rho \Phi^{-1}(u) \Phi^{-1}(v)+\left(\rho \Phi^{-1}(v)\right)^{2}\right)\right\} .
$$

Finally, the function $f(x, y)$ we are looking for, can be written as :

$$
f(x, y)=A f_{1}(x) f_{2}(y) \exp \left\{-\frac{\left(\rho^{2} x^{2}-2 \rho x y+\rho^{2} y^{2}\right)}{2\left(1-\rho^{2}\right)}\right\}
$$

where $\Phi^{-1}(u)=x$ and $\Phi^{-1}(v)=y$.

Figure 3 presents CopBP reconstructions obtained using this Gaussian copula. We see the interest of such an approach compared to standard BP.

The particular reconstruction (23) is parametrized by the correlation coefficient $\rho$ which is an hyperparameter of the reconstruction process. With a value $\rho=0$, that is with no correlations, the CopBP method reduces to the multiplicative MBP method. The specification of $\rho$ corresponds to the encoding of some prior information in the reconstruction procedure which helps to improve the quality of the reconstruction. For example, from physical or physiological knowledge, or from the experimental setting, the general orientation of the underlying object is known. Another situation is the case where a mean template for the object is available, for example as a result of previous experiments.

The hyperparameter $\rho$ may also be estimated from additional data. For instance, using some additional measurements, e.g. a third (may be partial) projection, it is easy to select the best value of $\rho$ which minimizes the distance between the actual projection and the one computed according to the model.

The general incorporation of prior information or additional data, with the automatic determination of the hyperparameters is a work in progress which is out of the scope of this Letter. What we want to emphasize through this simple example is the interest of the CopBP approach for including a such simple prior as the main orientation of the object, that leads to an noticeable improvement of the reconstruction. This suggests that copulabased approaches have a potential in the field of image reconstruction from projections. 

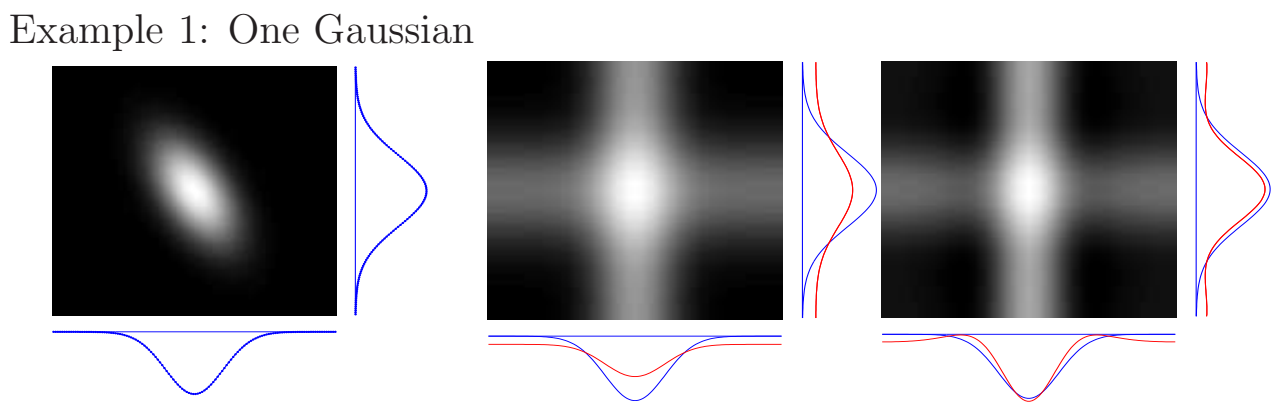

Original 1
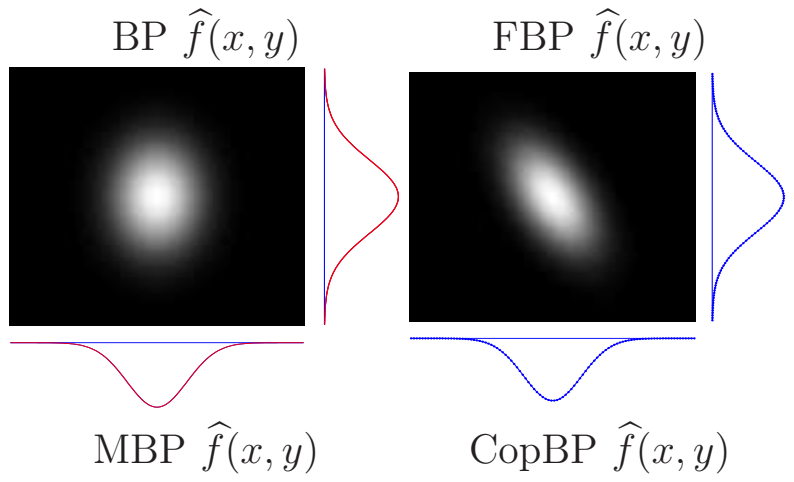

Example 2: Four Gaussians

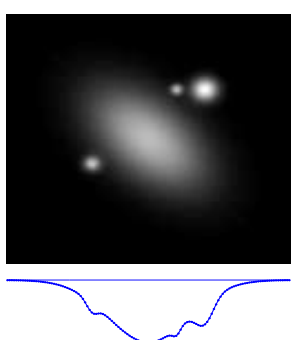

Original 2

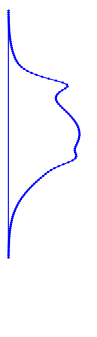

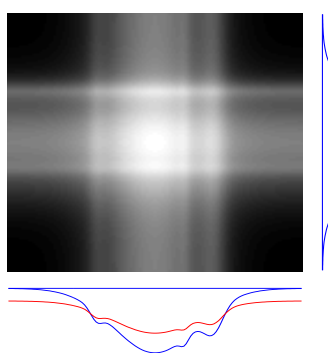

BP $\widehat{f}(x, y)$

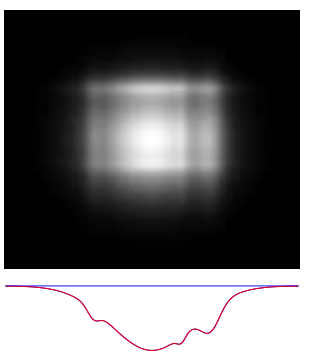

$$
\underset{10}{\operatorname{MBP}} \widehat{f}(x, y)
$$

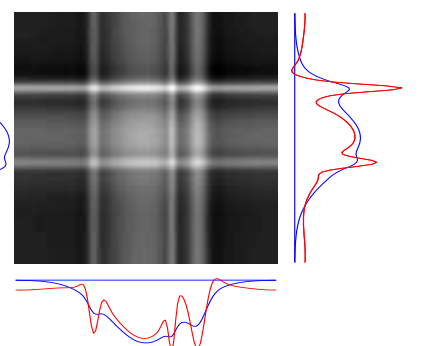

$\operatorname{FBP} \widehat{f}(x, y)$

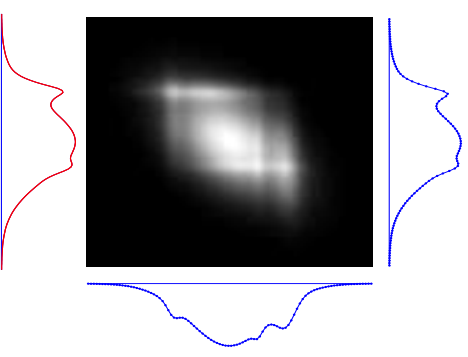

CopBP $\widehat{f}(x, y)$

Figure 3: Comparison between BP, FBP, MBP and CopBP on two synthetic examples. This shows the improvement obtained with MBP and CopBP methods compared to standard Back Projection (BP) or Filtered Back Projections (FBP). It is noted that marginals of the $\mathrm{BP}$ and $\mathrm{FBP}$ reconstructions differ from the original data while marginals of MBP/CopBP perfectly agree with initial data. 


\section{Maximum Entropy Copulas}

The selection of a particular copula is a difficult task. We propose here to look at this ill-posed inverse problem using the maximum entropy (ME) method. The principle of ME was first expounded by E.T. Jaynes in two seminal papers in $1957([23,24])$. It is the way to assign a probability distribution to a quantity on which we have partial information. The classical ME problem is to assign a probability law to a quantity on which we only know a few moments. Here, the problem is a bit different, because the partial information we have is not in terms of moments but in the form of the following constraints:

$$
\left\{\begin{array}{l}
C_{1}: \int f(x, y) d y=f_{1}(x), \quad \forall x \\
C_{2}: \int f(x, y) d x=f_{2}(y), \quad \forall y \\
C_{3}: \iint f(x, y) d x d y=1 .
\end{array}\right.
$$

Hence, the goal is to find the most general copula, in the ME sense, compatible with available information, that is, with the marginals/projections at hands.

\subsection{Problem's formulation}

Among all possible $f(x, y)$ satisfying the constraints (24) choose the one which optimizes a criterion $J(f)$, i.e :

$$
\hat{f}:=\operatorname{maximize}\{J(f)\} \text { subject to }(24) .
$$

Since the constraints are linear, if we choose a criterion which is a concave function, then there is a unique solution to the problem. Many entropies functional can serve as an objective function, e.g. [25-30] :
1. $J_{1}(f)=-\iint|f(x, y)|^{2} d x d y$, (-Energy or $L_{2}$-norm)
2. $J_{2}(f)=-\iint f(x, y) \ln f(x, y) d x d y$, (Shannon Entropy)
3. $J_{3}(f)=\iint \ln f(x, y) d x d y, \quad$ (Burg Entropy)
4. $J_{4}(f)=\frac{1}{1-\alpha}\left(1-\iint f^{\alpha}(x, y) d x d y\right), \quad$ (Tsallis Entropy)
5. $J_{5}(f)=\frac{1}{1-\alpha} \ln \iint f^{\alpha}(x, y) d x d y, \quad$ (Rényi Entropy). 
Our main contribution here is to find the generic expression for the solution of these criteria. The main tool is the classical Lagrange multipliers technique which consists in defining the Lagrangian functional

$$
\begin{aligned}
\mathcal{L}_{g}\left(f, \lambda_{0}, \lambda_{1}, \lambda_{2}\right) & =J(f)+\lambda_{0}\left(1-\iint f(x, y) d x d y\right) \\
& +\int \lambda_{1}(x)\left(f_{1}(x)-\int f(x, y) d y\right) d x \\
& +\int \lambda_{2}(y)\left(f_{2}(y)-\int f(x, y) d x\right) d y
\end{aligned}
$$

and find its stationnary point which is defined as the solution of the following system of equations:

$$
\left\{\begin{array}{l}
\frac{\partial \mathcal{L}_{g}\left(f, \lambda_{0}, \lambda_{1}, \lambda_{2}\right)}{\partial f}=0 \\
\frac{\partial \mathcal{L}_{g}\left(f, \lambda_{0}, \lambda_{1}, \lambda_{2}\right)}{\partial \lambda_{i}}=0
\end{array}\right.
$$

Here, we give the final expression, assuming that the integrals converge:

1. $\hat{f}(x, y)=-\frac{1}{2}\left(\lambda_{1}(x)+\lambda_{2}(y)+\lambda_{0}\right),($-Energy $)$

2. $\hat{f}(x, y)=\exp \left(-\lambda_{1}(x)-\lambda_{2}(y)-\lambda_{0}\right)$, (Shannon entropy)

3. $\hat{f}(x, y)=\frac{1}{\lambda_{1}(x)+\lambda_{2}(y)+\lambda_{0}}$, (Burg entropy)

4. $\hat{f}(x, y)=\frac{1-\alpha}{\alpha}\left(\lambda_{1}(x)+\lambda_{2}(y)+\lambda_{0}\right)^{\frac{1}{\alpha-1}}$, (Tsallis and Renyi entropies).

Where $\lambda_{1}(x), \lambda_{2}(y)$ and $\lambda_{0}$ are obtained by replacing these expressions in the constraints (24) and solving the resulting system of equations. When solving the Lagrangian functional equation which is concave in $f$, we assume that there exists a feasible $f>0$ with finite entropy. The results for Tsallis and Renyi entropies leads to the same family of distribution depending on $\alpha$ due to the monotonicity property of the logarithm function. For the two criteria -Energy and Shannon entropy, we can find analytical solutions for $\lambda_{1}(x), \lambda_{2}(y)$ and $\lambda_{0}$. For -Energy, we obtain:

$\lambda_{1}(x)=-2 f_{1}(x)+\int \lambda_{1}(x) \mathrm{d} x+2, \lambda_{2}(y)=-2 f_{2}(y)+\int \lambda_{2}(y) \mathrm{d} y+2$ and $\lambda_{0}=-2-\int \lambda_{1}(x) \mathrm{d} x-\int \lambda_{2}(y) \mathrm{d} y$, which finally gives: 


$$
\hat{f}(x, y)=f_{1}(x)+f_{2}(y)-1 .
$$

This is nothing else but the standard Backprojection mechanism (up to scale factor and a constant). Hence, the Backprojection method can be easily interpreted as a minimum norm solution. For the Shannon entropy, we get:

$$
\begin{gathered}
\lambda_{1}(x)=-\ln \left(f_{1}(x) \int \lambda_{1}(x) \mathrm{d} x\right), \lambda_{2}(y)=-\ln \left(f_{2}(y) \int \lambda_{2}(y) \mathrm{d} y\right) \text { and } \\
\lambda_{0}=\ln \left(\int \lambda_{1}(x) \mathrm{d} x \int \lambda_{2}(y) \mathrm{d} y\right) \text { which yields } \\
\hat{f}(x, y)=f_{1}(x) f_{2}(y) .
\end{gathered}
$$

This is now the MBP we obtained as associate to an independent copula. Unfortunately, in the cases of Burg, Tsallis and Renyi entropies, it is not possible to find analytical expressions for $\lambda_{0}, \lambda_{1}$, and $\lambda_{2}$ as functions of $f_{1}$ and $f_{2}$. Consequently a numerical approach is required, see for example [31].

Using equation (22) one can write all entropies in terms of copulas. For example, if we denote the Shannon entropy by $H(x, y)$ and the copula entropy by $H_{c}(u, v)$, then :

$$
H(x, y)=H(x)+H(y)+H_{c}(u, v) .
$$

The previous relation shows that the Shannon entropy of the bivariate distribution is the sum of the entropies provided by each marginal density and the copula entropy. In Appendix, we provide the proof of this result in the multivariate case, which is, to the best of our knowledge, original. This result shall be of interest for multidimensional tomography, especially 3D tomography. Therefore, maximizing the joint entropy, given the marginals, is equivalent to maximize the entropy of the copula $H_{c}(u, v)$. Since we only have here a domain constraint -the copula is defined on $[0,1]^{2}$-, the Shannon Maximum entropy copula is uniform, $c(u, v)=1$, and we obtain the MBP reconstruction (26). Now, if we look for a Shannon maximum entropy copula with an additional correlation constraint-that is we fix the correlation of the underlying normalized random variables-, then we end with a Gaussian copula, which in turn, lead us to the CopBP method with a Gaussian copula (22). Along these lines, it seems possible to characterize the different families of copula as maximum entropy solutions, possibly incorporating more prior information. More generally, it will also be interesting to characterize the copulas corresponding to the Burg/Rényi ME solutions.

Some simulations are reported Figure 3. The aim of these simulations from our Copula-Tomography package (which can be downloaded from [32]) is to illustrate the link between copula in tomography in the case of only two 
projections. The original 1 image simulated is a Gaussian and the original 2 image is formed by four Gaussians. We performed BP, FBP, MBP and CopBP on these images. We observe that for the MBP and the CopBP, the two projections on the reconstructed images match those from the original images which is not the case for the BP and the FBP.

\section{Conclusion}

The main contribution of this paper is to highlight a link between the notion of copulas in statistics and X-ray CT for small number of projections. This link brings up possible new approaches for image reconstruction in CT. We first presented the bivariate copulas and the image reconstruction problem in CT. We highlight the connexion between the two problems that consist in i) determining a joint bivariate pdf from its two marginals and ii) the CT image reconstruction from only two horizontal and vertical projections. We emphasize that in both cases, we have the same inverse problem for the determination of a bivariate function (an image) from the line integrals. We have indicated the potential of copula-based reconstruction methods, introducing the MBP (Multiplicative Back Projection) and CopBP (Copula Back Projection) methods. Current work addresses the characterization of family of copulas as well as the estimation of copulas parameters in the reconstruction process. We also intend to improve the results by accounting for more projections in the method, while keeping the copula approach.

\section{Appendix A. Relation with Shannon entropy in high dimension}

From the $n$-dimensional version of Sklar's theorem $[1,16]$, we have

$$
F\left(x_{1}, \ldots, x_{n}\right)=C\left(F_{1}\left(x_{1}\right), \ldots, F_{n}\left(x_{n}\right)\right) .
$$

Now taking the partial derivative in Eq.(A.1), since $u_{i}=F_{i}\left(x_{i}\right)$ it follows that the probability density function can be expressed by

$$
f\left(x_{1}, \ldots, x_{n}\right)=c\left(u_{1}, \ldots, u_{n}\right) \prod_{i=1}^{n} f_{i}\left(x_{i}\right) .
$$

Notice also that the differentials $d u_{i}=d F_{i}\left(x_{i}\right)=f_{i}\left(x_{i}\right) d x_{i}$,

and $\mathbf{d} \mathbf{x}=\prod_{i=1}^{n} d x_{i}$. Hence $\mathbf{d} \mathbf{u}=\prod_{i=1}^{n} f_{i}\left(x_{i}\right) d x_{i}$, and we remark that

$$
\int_{I^{n-1}} c(\mathbf{u}) \prod_{\substack{j=1 \\ j \neq i}}^{n} d u_{j}=\int_{\mathbb{R}^{n-1}} \frac{f\left(x_{1}, \ldots, x_{n}\right)}{f_{i}\left(x_{i}\right)} \prod_{\substack{j=1 \\ j \neq i}}^{n} d x_{j}=\frac{f_{i}\left(x_{i}\right)}{f_{i}\left(x_{i}\right)}=1 .
$$


Proof.

$$
\begin{aligned}
& H(\mathbf{x})=-\int_{\mathbb{R}^{n}}\left(c(\mathbf{u}) \prod_{i=1}^{n} f_{i}\left(x_{i}\right)\right) \ln \left(c(\mathbf{u}) \prod_{i=1}^{n} f_{i}\left(x_{i}\right)\right) \mathbf{d} \mathbf{x} \\
& =-\int_{\mathbb{R}^{n}}\left(c(\mathbf{u}) \prod_{i=1}^{n} f_{i}\left(x_{i}\right)\right)\left(\sum_{i=1}^{n} \ln f_{i}\left(x_{i}\right)\right) \prod_{i=1}^{n} d x_{i}-\int_{\mathbb{R}^{n}} c(\mathbf{u}) \ln c(\mathbf{u}) \prod_{i=1}^{n} f_{i}\left(x_{i}\right) d x_{i} \\
& =-\sum_{i=1}^{n} \int_{\mathbb{R}^{n}}\left(c(\mathbf{u}) \prod_{\substack{j=1 \\
j \neq i}}^{n} f_{j}\left(x_{j}\right) d x_{j}\right) f_{i}\left(x_{i}\right) \ln f_{i}\left(x_{i}\right) d x_{i}-\int_{I^{n}} c(\mathbf{u}) \ln c(\mathbf{u}) \mathbf{d} \mathbf{u} \\
& =-\sum_{i=1}^{n}\left(\int_{I^{n-1}} c(\mathbf{u}) \prod_{\substack{j=1 \\
j \neq i}}^{n} d u_{j}\right)\left(\int_{\mathbb{R}} f_{i}\left(x_{i}\right) \ln f_{i}\left(x_{i}\right) d x_{i}\right)+H_{c}(\mathbf{u}) \\
& =-\sum_{i=1}^{n} \int_{\mathbb{R}} f_{i}\left(x_{i}\right) \ln f_{i}\left(x_{i}\right) d x_{i}+H_{c}(\mathbf{u}) \\
& =\sum_{i=1}^{n} H\left(x_{i}\right)+H_{c}(\mathbf{u}) \text {. }
\end{aligned}
$$

Eq.(A.3) shows that the entropy $H(\mathbf{x})=-\int_{\mathbb{R}^{n}} f(\mathbf{x}) \ln f(\mathbf{x}) d \mathbf{x}$ of the joint multivariate distribution is the sum of the entropies provide by each marginal density $H\left(x_{i}\right)$ and the copula entropy $H_{c}(\mathbf{u})$.

\section{References}

[1] A. Sklar, Fonctions de repartition à n dimensions et leurs marges, Publications de l'Institut de Statistique de L'Université de Paris 8 (1959) $229-231$.

[2] H. Joe, Multivariate extreme-value distributions with applications to environmental data, The Canadian Journal of Statistics 22 (1994) 4764 . 
[3] C. Genest, A.-C. Favre, Everything you always wanted to know about copula modeling but were afraid to ask, Journal of Hydrologic Engineering 12 (2007) 347-368.

[4] J. Kim, Y. Jung, E. Sungur, K. Han, C. Park, I. Sohn, A copula method for modeling directional dependence of genes, BMC bioinformatics 9 (1) (2008) 225.

[5] D. Zhang, T. Martin, L. Peng, Nonparametric estimation of the dependence function for a multivariate extreme value distribution, Journal of Multivariate Analysis 99 (2006) 577-588.

[6] W. C. Kallenberg, Modelling dependence, Insurance: Mathematics and Economics 42 (2008) 127-146.

[7] H. Joe, Multivariate Models and Dependence Concepts, London: Chapman \& Hall, 1997.

[8] J. Hadamard, Sur les problèmes aux dérivées partielles et leur signification physique, Princeton University Bulletin 13 (1902) 49-52.

[9] A. Tarantola, Inverse Problem Theory and Methods for Model Parameter Estimation, 1st Edition, SIAM: Society for Industrial and Applied Mathematics, 2004.

[10] J. Idier, Bayesian Approach to Inverse Problems, 1st Edition, WileyISTE, 2008.

[11] H. Pan, Z.-P. Liang, T. S. Huang, Estimation of the joint probability of multisensory signals, Pattern Recognition Letters 22 (13) (2001) 14311437.

[12] J. Radon, Über die bestimmung von funktionen durch ihre integralwerte längs gewisser mannigfaltigkeiten, Ber. Verh. Säch. Akad. Wiss. Leipzig, Math. Nat. Kl 69 (1917) 262-277.

[13] J. Radon, On the determination of functions from their integral values along certain manifolds, IEEE transactions on medical imaging 5 (4) (1986) 170-176.

[14] A. M. Cormack, Representation of a function by its line integrals with some radiological application, J. Appl. Physics 34 (1963) 2722-2727.

[15] R. Nelsen, An introduction to copulas, Springer Verlag, 2006. 
[16] B. Schweizer, A. Sklar, Probabilistic Metric Spaces, North Holland New York, 1983.

[17] S. Deans, The Radon transform and some of its applications, A WileyInterscience Publication, New York, 1983.

[18] A. Kak, M. Slaney, Principles of Computerized Tomographic Imaging, Society of Industrial and Applied Mathematics, 1988.

[19] F. Natterer, The mathematics of computerized tomography, Society for Industrial Mathematics, 2001.

[20] A. Markoe, Analytic Tomography, Cambridge University Press, 2006.

[21] G. Herman, A. Kuba, S. O. service, Advances in discrete tomography and its applications, Birkhäuser, 2007.

[22] D.-B. Pougaza, A. Mohammad-Djafari, J.-F. Bercher, Utilisation de la notion de copule en tomographie, in: XXIIe colloque GRETSI, Dijon, France, 2009.

[23] E. Jaynes, Information Theory and Statistical Mechanics, Physical Review 106 (4) (1957) 620-630.

[24] E. Jaynes, Information theory and statistical mechanics. II, Physical Review 108 (2) (1957) 171-190.

[25] C. Shannon, A mathematical theory of communication, Bell System Technical Journal 27 (1948) 432-379.

[26] A. Renyi, On measures of entropy and information, in: Proceedings of the 4th Berkeley Symposium on Mathematical Statistics and Probability, Vol. 1, 1961, pp. 547-561.

[27] A. Mohammad-Djafari, Jérôme Idier, Maximum Likelihood Estimation of the Lagrange Parameters of the Maximum Entropy Distributions, C.R. Smith, G.J. Erikson and P.O. Neudorfer Edition, Kluwer Academic Publ., 1991, pp. 131-140.

[28] J. Kapur, H. Kesavan, Entropy optimization principles with applications, Academic Press, Boston; Tokyo, 1992.

[29] E. Pasha, S. Mansoury, Determination of Maximum Entropy Multivariate Probability Distribution under some Constraints, Applied Mathematical Sciences 2 (57) (2008) 2843-2849. 
[30] D. Yu, L. Deng, A. Acero, Using continuous features in the maximum entropy model, Pattern Recognition Letters 30 (14) (2009) 1295-1300.

[31] A. Mohammad-Djafari, A Matlab Program to Calculate the Maximum Entropy Distributions, T.W. Grandy Edition, Kluwer Academic Publ., 1991, pp. 221-233.

[32] D.-B. Pougaza, A. Mohammad-Djafari, Copula-Tomography Package, http://users.aims.ac.za/ doriano. 